\title{
Estimating demand for new products using a discrete price variable
}

Received (in revised form): 4th January, 2008

\section{Randall C. Campbell}

is an assistant professor of economics at Mississippi State University. He obtained his PhD in Economics at Louisiana State University in 1999 and his primary research and teaching interests are in applied econometrics. He previously worked as an economist in the market research department at Southwestern Bell, and has published articles in both marketing and economics journals.

\section{Keywords discrete explanatory variables, demand estimation, probit, new products}

\begin{abstract}
We conduct a set of Monte Carlo sampling experiments to examine the use of discrete explanatory variables in market research for new products. We compare three alternative estimators on the basis of mean square error. As part of the experimental design we vary the number of price points and the distribution of the error term. In addition, we consider demand functions that are linear, linear with structural breaks and nonlinear. In each case, our results show that using two discrete price points leads to the lowest estimation risk.
\end{abstract}

Journal of Targeting, Measurement and Analysis for Marketing (2008) 16, 115-121. doi:10.1057/jt.2008.1; published online 10 March 2008

\section{INTRODUCTION}

This paper examines the use of discrete price variables in market research for new products. When estimating demand for new products market researchers often conduct surveys to ask respondents about expected future demand. How consumers respond to price changes is of particular interest to market researchers who want to determine what price to charge for the product when it goes on the market. As Wyner ${ }^{1}$ explains, it is common for market researchers to use a set of discrete price points to estimate market demand. Independent samples of customers are asked about expected purchases at a single price. For example, suppose a firm is introducing a new product that they plan to price somewhere between $\$ 5$ and $\$ 20$ and the firm decides to conduct a survey on 400 respondents. The survey might have four versions with price points of $\$ 5, \$ 10, \$ 15$ and

Correspondence: Randall C. Campbell, Department of Finance and Economics, Mississippi State University, Mississippi State, MS 39762, USA. Tel: +1 6623251516 ;

Fax: +1 6623251977 ;

E-mail: rcampbell@cobilan.msstate.edu
\$20. One hundred respondents would be asked how much they would purchase if the price were $\$ 5,100$ respondents would be asked at a price of $\$ 10$ and so forth.

Klein and Sherman ${ }^{2,3}$ consider the problem of response bias in survey data for new product demand. Terza ${ }^{4}$ considers the problem of ordinal qualitative regressors in linear models. He uses the term 'ordinal qualitative regressor' to refer to a variable with arbitrary mean and variance and an unknown threshold, such as a bond rating. The term 'cardinal qualitative regressor' refers to a variable that is ordered, but which has known category thresholds. For example, in market research it is common to report continuous variables such as income or age as categorical variables. Hsiao and Mountain ${ }^{5}$ and Kukuk ${ }^{6}$ consider the problem of cardinal qualitative regressors in market research.

The problem we consider is similar to that of cardinal qualitative regressors in that while price is a continuous variable, we only obtain observations for a discrete ordered set of values. We, however, consider a case in which the price 
points represent an actual price value. For the cardinal qualitative regressors, such as income, we do not actually observe the respondent's true income, which leads to biased estimation. Since the price points represent an actual value our estimator is unbiased.

We conduct a set of Monte Carlo sampling experiments to examine the use of discrete price variables in market research for new products. We are interested in how well one can estimate price elasticities using discrete price points and the optimal number of price points a researcher should use. Intuitively, one might expect to obtain better estimates and forecasts the more prices we observe data for, since a greater number of price points more closely reflects the continuous nature of the price variable. We consider estimation in a linear model, a linear model with a structural break and a binary choice model. As part of the experimental design we vary the number of price points and the distribution of the errors. We compare alternative estimators based on the mean square error (MSE) in our sampling experiments as well as on prediction error, both in- and out-of-sample. The next section presents our demand model and discusses the alternative model formulations. The subsequent section presents Monte Carlo estimation results while the last section concludes the paper.

\section{DEMAND MODELS FOR NEW PRODUCTS}

We consider models in which a firm wants to estimate demand for a new product that has not yet been priced. The firm would typically conduct a telephone survey in which they describe the product, ask respondents how many units they would purchase at a given price, and collect some demographic information about the respondent. We carry out Monte Carlo experiments examining the empirical risk of estimators under both discrete and continuous price variables. We consider a linear model, a linear model with a structural break and a probit model. The experimental designs for each model are given below.

\section{Linear model}

We first estimate a linear regression model of the form

$$
y=X \beta+e
$$

where $y$ is the $T \times 1$ vector of sample observations on the dependent variable, $X$ is the $T \times K$ matrix of explanatory variables, $e$ is the $T \times 1$ vector of unknown errors and $\beta$ is the $K \times 1$ vector of unknown parameters.

We generate data using the true demand model

$$
y_{t}=80-2 \cdot P_{t}+3 \cdot I N C_{t}-0.2 \cdot A G E_{t}+e
$$

where $y_{t}$ is the quantity the respondent plans to purchase, $P_{t}$ is the price of the product, $I N C_{t}$ is the respondent's income and $A G E_{t}$ is the respondent's age. In our Monte Carlo experiments, $I N C_{t}$ is a random variable between 15 and 100 (measured in $\$ 1000$ 's), $A G E_{t}$ is a random integer between 20 and 80, and random errors are drawn from a $N\left(0, \sigma^{2}=25\right)$ population. ${ }^{5-7}$

In market research for new products, respondents are generally asked how much of a product they would purchase at one of $J$ price points, which are randomly rotated among the respondents. Thus, we generate data using $2,3,6$, 9 and 21 discrete price points in our Monte Carlo experiments. For comparison we also generate data using a continuous price variable. During each of $N=1,000$ Monte Carlo iterations, we generate the dependent variable as $y=X_{a} \beta+e$. In addition, we generate a hold out sample as $y_{0}=X_{a 0} \beta+e_{0}$, which we use to examine outof-sample prediction. We use an estimation sample size of $T=100$ and a hold out sample of size $T_{0}=50$ for all experiments. Given the constant sample size, an increase in the number of price points means that fewer respondents will be tested at each price point.

The unbiasedness of the ordinary least squares (OLS) estimator does not depend on the range or variation of $X$. The variance of the OLS estimator, however, is inversely related to the variation in $X$ about its mean. ${ }^{8-11}$

In our example, the variance of the OLS estimator for the price coefficient is given by $\operatorname{var}\left(b_{p}\right)=\sigma^{2} / \Sigma_{i=1}^{\mathrm{T}}\left(p_{t}-\bar{p}\right)^{2}$. Thus, an estimator based 
on fewer price points results in an unbiased estimator with a smaller variance than an estimator based on more price points, holding everything else constant.

Consider two vectors of price points, $P_{1}=[2040]^{\prime}$ and $P_{2}=\left[\begin{array}{lll}20 & 30 & 40\end{array}\right]^{\prime}$ and a sample size of $T=30$.

Assuming an equal number of observations at each price point, we would have 15 observations for each price point in vector $P_{1}$ and 10 observations for each price point in vector $P_{2}$. Note that both price vectors have a mean of 30 . For sample 1 :

$$
\Sigma_{i=1}^{\mathrm{T}}\left(p_{t}-\bar{p}\right)^{2}=15(20-30)^{2}+15(40
$$

$-30)^{2}=3,000$, while for sample $2: \Sigma_{i=1}^{\mathrm{T}}\left(p_{t}-\bar{p}\right)^{2}=$ $10(20-30)^{2}+10(30-30)^{2}+10(40-30)^{2}=2,000$.

Thus, given a constant sample size, $P_{1}$ results in a more precise estimator. ${ }^{12}$ This is contrary to the intuition that having data for a greater number of price points should result in a better estimator. Monte Carlo results for this set of experiments are given in the Results section under Linear model.

\section{Linear model with a structural break}

We next estimate a linear model with a structural break. We assume that the structural break occurs at some price level. In this case, we assume that demand becomes more elastic when the price is greater than $\$ 30$. The true demand model is given by

$y_{t}=\left\{\begin{array}{cc}80-2 \cdot P_{t}+3 \cdot I N C_{t}-0.2 \cdot A G E_{t}+e, & P<30 \\ 95-2.5 \cdot P_{t}+3 \cdot I N C_{t}-0.2 \cdot A G E_{t}+e, & P \geqslant 30\end{array}\right.$

Here we expect that the estimator based on a larger number of price points will perform better than an estimator based on a smaller number of price points since the larger number of price points includes observations around the point where the break occurs. ${ }^{13}$

The price, income and age variables and the error term are defined as in the linear model. Using dummy variables we estimate the following model:

$$
\begin{aligned}
y_{t}= & \beta_{1}+\delta_{1} \cdot D_{t}+\beta_{2} \cdot P_{t}+\delta_{2} \cdot\left(P_{t} \cdot D_{t}\right) \\
& +\beta_{3} \cdot I N C_{t}+\beta_{4} \cdot A G E_{t}+e
\end{aligned}
$$

We use an F-test to test the null hypothesis $H_{0}: \delta_{1}=\delta_{2}=0$. It seems reasonable that the greater the number of price points, the more likely we are to detect the structural change and thus we should reject $H_{0}$ more often. Monte Carlo results for this set of experiments are given in the Results section under Linear model with a structural break.

\section{Binary choice model}

Often in market research the question is whether or not a consumer will buy a product rather than how much the consumer will buy. Therefore, we consider a binary choice model in this section. For this model, we generate data following:

$$
y_{t}^{*}=-0.2-0.15 \cdot P_{t}+0.12 \cdot I N C_{t}-0.05 \cdot A G E_{t}+e_{t}
$$

where $y_{t}^{\star}$ is an unobserved latent variable. This yields the observable binary variable as

$$
y_{t}= \begin{cases}1, & \text { if } y_{t}^{*}>0 \\ 0, & \text { if } y_{t}^{*} \leqslant 0\end{cases}
$$

The price, income and age variables are defined as before. In the binary choice model we, however, draw errors from a $N\left(0, \sigma^{2}=1\right)$ population. Since we assume normal errors we use probit to estimate the binary choice model. In the probit model, the covariance matrix is the negative inverse of the Hessian. In this case, it is not clear whether the standard errors of the estimator increase or decrease with the variation in the explanatory variables about their means. Results for this set of Monte Carlo experiments are given in the next section under Binary choice model.

\section{RESULTS}

In this section, we present results from our Monte Carlo experiments. For nearly all of the experiments we find that MSE is lower the fewer the number of price points, holding everything else constant. Since the variance of the least squares estimator is inversely related to the variation in each explanatory variable about its mean, we expected this to be true for the linear regression model. We, however, did not expect that it would hold in the other models since this result is not consistent with our intuition that using more price information should result in better estimates of the price coefficient. The 
results for the various models are given in the following three sections.

\section{Linear model}

As stated above, it can be shown that using fewer price points, holding the total number of observations constant, will lead to lower MSE in the linear regression model since the variance of the OLS estimator is inversely related to the variation in each regressor (although intuitively one might expect more price points to lead to more precise estimates, this is not the case). The OLS estimator is unbiased regardless of the number of price points used in collecting the data. Since the variance of the OLS estimator is smaller when fewer price points are used the MSE will be lower for fewer price points.

We consider 2, 3, 6, 9 and 21 price points as well as a continuous price variable. In addition, we vary the maximum and minimum prices used. The wider the price range used, the smaller the variance for the OLS estimator. The price ranges are given in Table 1 . The average price is $\$ 30$ for all experiments. For example, the price range between 28 and 32 corresponds to price points $P=\left[\begin{array}{ll}28 & 32\end{array}\right]^{\prime}$ for 2 price points, $P=\left[\begin{array}{lll}28 & 30 & 32\end{array}\right]^{\prime}$ for 3 price points, $P=\left[\begin{array}{llllll}28 & 28.8 & 29.6 & 30.4 & 31.2 & 32\end{array}\right]^{\prime}$ for 6 price points and so forth. Table 1 gives the empirical root mean square error (RMSE) of the OLS estimates.

As expected, the results show that the RMSE decreases as we increase the price range. The empirical bias is nearly zero in each experiment and the variance decreases as we increase the price range. The RMSE increases as we increase the number of price points and is greatest when the price variable is continuous. This occurs because the variance of price about its mean decreases as the number of price points increases. To examine whether there is some cost associated with using fewer price points we examine prediction error. Table 2 gives the root mean square prediction error, both in- and out-ofsample.

In the linear regression model, the prediction error does not vary with the price range. Prediction error does vary slightly with the number of price points, but there is no consistent pattern. We find the smallest in-sample prediction error with 3 price points, and the smallest outof-sample prediction error with 2 price points. Based on these results, the best approach for market research would be to pick the highest and lowest prices the firm would consider charging for the product and use just these 2 price points in the survey.

\section{Linear model with a structural break}

We expect that a larger number of price points might perform better when there is a kinked demand curve. In this example, demand is more price elastic when price is greater than $\$ 30$. While having price observations near the centre of the price range does not seem to matter for the linear demand curve, we would expect that some values near $\$ 30$ are needed to detect the structural break. We find that this is partially true; we need at least 2 price points both above and below $\$ 30$ to obtain unique estimates. Thus, we

Table 1: RMSE of OLS estimator (linear model)

\begin{tabular}{lllllcc}
\hline Price range & 2 price points & 3 price points & 6 price points & 9 price points & 21 price points & Continuous price \\
\hline$[28,32]$ & 7.477 & 9.819 & 11.839 & 12.585 & 13.709 & 14.297 \\
{$[24,36]$} & 2.948 & 3.707 & 4.262 & 4.460 & 4.827 & 4.998 \\
{$[20,40]$} & 2.246 & 2.657 & 2.915 & 3.002 & 3.206 & 3.292 \\
{$[10,50]$} & 1.892 & 2.050 & 2.113 & 2.129 & 2.207 & 2.232 \\
\hline
\end{tabular}

Table 2: Prediction RMSE (linear model)

\begin{tabular}{lllllll}
\hline Price range & 2 price points & 3 price points & 6 price points & 9 price points & 21 price points & Continuous price \\
\hline In-sample & 49.1549 & 49.1400 & 49.1472 & 49.1462 & 49.1419 & 49.1443 \\
Hold-out & 36.3125 & 36.3567 & 36.3583 & 36.3461 & 36.3465 & 36.3452 \\
\hline
\end{tabular}


consider only 6,9 and 21 price points as well as a continuous price variable in this set of experiments. Table 3 gives the empirical RMSE of the OLS estimates. In addition, row two of Table 3 gives the number of times an F-test fails to reject the null hypothesis of no structural break out of 1,000 Monte Carlo trials for a price range of 28 to $32 .{ }^{14}$

We find a slight empirical bias for the OLS estimator in this set of experiments. The variance continues to increase with the number of price points (except for one case when we move from 6 to 9 price points with a price range from 10 to 50). The null hypothesis of no structural break is actually rejected more often when we use 6 or 9 price points than with a greater number of price points. It was rejected more often for 9 price points than for 6 , probably because the midpoint of $\$ 30$ is one of the prices used for an odd number of price points. Table 4 gives the root mean square prediction error, both in- and outof-sample. We again find no consistent pattern between the number of price points used and the prediction error. Thus, a market researcher who believes that there may be one (or more) structural breaks would probably want to use the minimum of 2 price points for each section of the demand curve. ${ }^{11,15}$

\section{Binary choice model}

We next examine a binary choice model, which is a common nonlinear model in market research. The binary choice model is used when customers are asked whether they would purchase a product or not. We chose the parameters such that there are approximately 50 per cent 'buy' and 50 per cent 'not buy' responses. Because the binary choice model is nonlinear we expect that a larger number of price points might give better results since 2 price points cannot detect the curvature. In addition, the probit estimator is not unbiased and so there may be a larger bias when fewer price points are used to generate the sample data. We compare the alternative data generating processes based on RMSE, prediction error, both in terms of the percentage of correct predictions and the RMSE of the predicted latent values. Since we assume normal errors we estimate the model using probit. Table 5 gives the empirical RMSE of the probit estimates.

Despite the nonlinearity of the data and the bias of the probit estimator, we still find an

Table 3: RMSE of OLS estimator (linear model with structural break)

\begin{tabular}{lcccc}
\hline Price range & $\mathbf{6}$ price points & $\mathbf{9}$ price points & 21 price points & Continuous price \\
\hline$[28,32]$ & 58.948 & 68.455 & 68.973 & 69.261 \\
Fail to reject & 346 & 318 & 441 & 451 \\
{$[24,36]$} & 19.555 & 21.793 & 22.486 & 22.933 \\
{$[20,40]$} & 11.792 & 12.572 & 13.295 & 13.761 \\
{$[10,50]$} & 6.213 & 5.946 & 6.646 & 7.083 \\
\hline
\end{tabular}

Table 4: Prediction RMSE (linear model with structural break)

\begin{tabular}{lllll}
\hline Price range & $\mathbf{6}$ price points & $\mathbf{9}$ price points & 21 price points & Continuous price \\
\hline In-sample & 48.6403 & 48.6250 & 48.6135 & 48.6383 \\
Hold-out & 36.6849 & 36.7101 & 36.6851 & 36.6902 \\
\hline
\end{tabular}

Table 5: RMSE of probit estimator (binary choice model)

\begin{tabular}{llcrrrr}
\hline Price range & $\mathbf{2}$ price points & $\mathbf{3}$ price points & $\mathbf{6}$ price points & $\mathbf{9}$ price points & $\mathbf{2 1}$ price points & $\begin{array}{l}\text { Continuous } \\
\text { price }\end{array}$ \\
\hline$[28,32]$ & 8.597 & 10.800 & 12.184 & 14.342 & 18.917 \\
{$[24,36]$} & 2.959 & 3.581 & 4.534 & 4.737 & 5.760 & 3.777 \\
{$[20,40]$} & 1.773 & 2.722 & 3.113 & 3.327 & 3.959 \\
{$[10,50]$} & 1.487 & 1.212 & 1.505 & 1.770 & 4.097 & 2.945 \\
\hline
\end{tabular}


increase in the RMSE of the probit estimates as we move from few price points to a continuous price variable. Although it is not shown in the table, the bias is lower when we use just 2 price points than for continuous price for all price ranges. Thus, we again obtain the result that ignoring the middle of the price range allows us to obtain estimates with both smaller bias and variance. Tables 6 and 7 give the root mean square prediction error for the latent values, both in- and out-of-sample. The tables show that in the probit model the prediction RMSE does change as the price range changes.

Although the prediction RMSE does not strictly increase or decrease with the number of price points, the continuous price models clearly perform worst in all cases. The models with relatively few $(2,3$ or 6$)$ price points predict the actual latent value best on average. We are, however, more interested in predicting whether a customer will buy the product or not. We classify those with predicted probabilities above 0.5 to be 'buyers' and those with predicted probabilities below 0.5 to be 'nonbuyers'. Based on this arbitrary cut-off rule Tables 8 and 9 give the percent of correct predictions, both in- and out-of-sample.

There is no discernible difference as we move across each row using more price points. We do note that as we move down each column it appears as though the fewer the number of price points, the more sensitive the estimator is to the price range.

Table 6: In-sample RMSE of predicted latent values (binary choice model)

\begin{tabular}{lllllrr}
\hline Price range & 2 price points & 3 price points & 6 price points & 9 price points & 21 price points & $\begin{array}{l}\text { Continuous } \\
\text { price }\end{array}$ \\
& & & & & & \\
\hline$[28,32]$ & 44.0160 & 39.7120 & 31.8136 & 35.5910 & 47.5391 & 71.3076 \\
{$[24,36]$} & 49.5893 & 43.2472 & 41.6139 & 44.5690 & 53.4819 & 74.8770 \\
{$[20,40]$} & 35.4637 & 51.9243 & 51.7090 & 41.6841 & 62.5761 & 71.8476 \\
{$[10,50]$} & 43.9325 & 32.7510 & 51.7363 & 55.2295 & 65.4226 & 102.0885 \\
\hline
\end{tabular}

Table 7: Out-of-sample RMSE of predicted latent values (binary choice model)

\begin{tabular}{lllllll}
\hline Price range & 2 price points & 3 price points & $\mathbf{6}$ price points & 9 price points & 21 price points & $\begin{array}{l}\text { Continuous } \\
\text { price }\end{array}$ \\
\hline$[28,32]$ & 28.1429 & 25.3787 & 20.7042 & 23.1043 & 30.5457 & 46.3621 \\
{$[24,36]$} & 33.6467 & 28.1704 & 27.1230 & 29.5860 & 34.6090 & 48.9786 \\
{$[20,40]$} & 24.8158 & 34.1239 & 33.6018 & 27.6548 & 40.8501 & 46.6744 \\
{$[10,50]$} & 32.7575 & 23.2676 & 36.7779 & 38.5645 & 43.6115 & 71.4642 \\
\hline
\end{tabular}

Table 8: Per cent correct predctions in-sample (binary choice model)

\begin{tabular}{lllllll}
\hline Price range & 2 price points & 3 price points & $\mathbf{6}$ price points & 9 price points & 21 price points & Continuous price \\
\hline$[28,32]$ & 94.22 & 94.50 & 94.33 & 94.32 & 94.27 & 94.28 \\
{$[24,36]$} & 94.54 & 95.00 & 94.49 & 94.58 & 94.47 & 94.49 \\
{$[20,40]$} & 94.75 & 95.10 & 94.48 & 94.75 & 94.54 & 94.59 \\
{$[10,50]$} & 92.95 & 93.30 & 93.74 & 94.75 & 94.65 & 94.67 \\
\hline
\end{tabular}

Table 9: Per cent correct predictions out-of-sample (binary choice model)

\begin{tabular}{lllllll}
\hline Price range & 2 price points & 3 price points & $\mathbf{6}$ price points & 9 price points & 21 price points & Continuous price \\
\hline$[28,32]$ & 88.23 & 87.82 & 87.66 & 87.49 & 87.55 & 87.48 \\
{$[24,36]$} & 90.54 & 88.86 & 88.23 & 88.38 & 88.45 & 88.42 \\
{$[20,40]$} & 92.29 & 89.99 & 88.99 & 89.35 & 89.14 & 89.02 \\
{$[10,50]$} & 94.45 & 91.81 & 93.59 & 93.10 & 92.87 & 92.33 \\
\hline
\end{tabular}




\section{CONCLUSIONS}

This paper examines the use of discrete price points in market research survey data for new products. Our goal is to provide a guideline for market researchers to determine the optimal number of price points to use in survey research or whether a continuous price variable (which could be simulated with a large number of price points) should be used. We carry out Monte Carlo experiments examining the use of discrete price points in several models including linear, linear with a structural break and binary choice models.

The results do not lead to a definite conclusion as to the optimal number of price points. While intuition suggests that using as many price points as is feasible for a given survey would provide better data and lead to better estimation results, our Monte Carlo experiments show the opposite to be true. In nearly all of our Monte Carlo experiments we find that using only 2 price points leads to lower estimation risk and better prediction, both in the estimation sample and in a hold out sample. In the linear regression model, this occurs because the variance of the OLS estimator is inversely related to the variation of the explanatory variables about their means. This result, however, does not explain why fewer price points lead to better performance in the other models where one might expect more price points to provide better information and thus better estimates.

Future research should consider alternative error distributions. While the conclusion that fewer price points leads to better estimation is robust in the models we examined, this may not hold for nonnormal errors or mixture models. Based on this analysis, our recommendation for market researchers is to utilise just 2 price points, the maximum and minimum prices the firm would be willing to charge for the product. Using fewer price points provides better estimates for the models we examined and has the advantage of lowering survey costs.

\section{Acknowledgments}

I thank an anonymous referee for helpful comments and suggestions. I am responsible for any errors that remain.

\section{References and Notes}

1 Wyner, G. A. (1993) 'Customer-based pricing research', Marketing Research, Vol. 5, pp. 50-52.

2 Klein, R. and Sherman, R. (1997) 'Estimating new product demand from biased survey data', Journal of Econometrics, Vol. 76, pp. 53-76.

3 Klein, R. and Sherman, R. (2002) 'Shift restrictions and semiparametric estimation in ordered response models', Econometrica, Vol. 70, pp. 663-691.

4 Terza, J. V. (1987) 'Estimating linear models with ordinal qualitative regressors', Journal of Econometrics, Vol. 34, pp. 275-291.

5 Hsiao, C. and Mountain, D. (1985) 'Estimating the short-run income elasticity of demand for electricity by using crosssectional categorized data', Journal of the American Statistical Association, Vol. 80, pp. 259-265.

6 Kukuk, M. (2002) 'Indirect estimation of (latent) linear models with ordinal regressors', Statistical Papers, Vol. 43, pp. 379-399.

7 For simplicity we assume that actual income and age are used. In general these data are collected as categorical variables as discussed by Hsiao and Mountain ${ }^{5}$ and Kukuk ${ }^{6}$.

8 See, for example, Kennedy ${ }^{9}$ (p. 57), Hill et al. ${ }^{10}$ (p. 116) and Greene $^{11}$ (p. 135).

9 Kennedy, P. (2003) 'A Guide to Econometrics', MIT Press, Cambridge, MA.

10 Hill, R. C., Griffiths, W. E. and Lim, G. C. (2008) 'Principles of Econometrics', John Wiley and Sons, Hoboken, NJ.

11 Greene, W. H. (2008) 'Econometric Analysis', Prentice-Hall, Upper Saddle River, NJ.

12 A reviewer correctly noted that if we simply added 15 observations to the $\$ 30$ price point in $P_{2}$, there would be no change in variance. Adding observations is, however, costly for a firm. In addition, we would expect lower, not equal, risk when collecting more data. In all experiments, we assume a constant number of surveys while varying the number of customers surveyed at each price.

13 We cannot obtain unique estimates for this model with fewer than four price points since we need at least two different prices in each subset.

14 The null hypothesis $H_{0}: \delta_{1}=\delta_{2}=0$ was rejected in every trial when the price range was $24-36$ or wider.

15 We estimate a simple linear model with a structural break following the methodology in Greene ${ }^{11}$ (pp. 120-128). An interesting area for future research is to estimate a set of latent class models. In addition to estimating the unknown parameters, one could examine whether the latent class model correctly identifies where the break occurs, predict the number of classes, and predict class membership. In addition, the variance among different subgroups could be changed in the latent-class model. Thus, the latent-class model would be a valuable extension in this area of research. 